2) Incubation time of 5 minutes was enough and proper in aspect of less enzyme leakage.

3) Urea showd fast-moving isozyme and lactate showed slow-moving isozyme in tissue section as proved by agar gel electrophoresis.

4) Various tissues constructing the rat organs were suggested to have their own isozyme patterns by this method.

Acknowledgement is made to Prof. Y. Shimazono, Department of Biochemistry of this University, for the technical instruction about agar gel electrophoresis.

\title{
References
}

1) Allen, J.M. : Ann. N.Y. Acad. $94: 937,1961 \quad$ 2) Brody, I.A. : Nature $201: 685,1964 \quad 3$ ) Brody, I.A. and Engel, W.K. : J. Histochem. Cytochem. 12:687, 1964 4) Ressler, N., Joseph, R. and Shulz. J.:J. Lab. Clin. Med. $60: 349,1962$ 5) Fahimi, H.D. and Amarsingham, C.R.: J. Cell Biol. $22: 29,1964$

\section{Study of Substrate Specificity and Co-Factors in the Histochemical Staining of $3 \beta$-Hydroxysteroid Dehydrogenase}

\author{
Hisao Takayasu, Yoshio Aso, Kenji Kinoshita, \\ Yoshinobu Hoshino, and Isao MURAHASHI, \\ Department of Urology, Faculty of Medicine, University of Tokyo, Tokyo.
}

In staining $3 \beta$-hydroxy dehydrogenase, cholesterol, pregnenolone, $17 \alpha$ hydroxy pregnenolone, dehydroepiandrosterone and epiandrosterone were used as substeates.

As materials, the fresh-frozen sections and the soft-paraffin embedded sections after cold-acetone fixation of the adrenal glands, testis, prostate, kidney and liver were stained for this enzyme.

The degrees of formazan formation were different depending upon different substrates and organs. Epiandrosterone having no double bond in the position $5\left(\Delta^{5}\right)$ was proved to be a substrate for this enzyme in the adrenal gland. (Fig. 1, 2)

TPN, DPN and DPNH were investigated for their effects as co-factor.

It was shown that TPN or DPN was necessary in staining this enzyme. The soft-paraffin embedded sections after cold-acetone fixation did not produce formazan when DPN was used as a co-factor. 


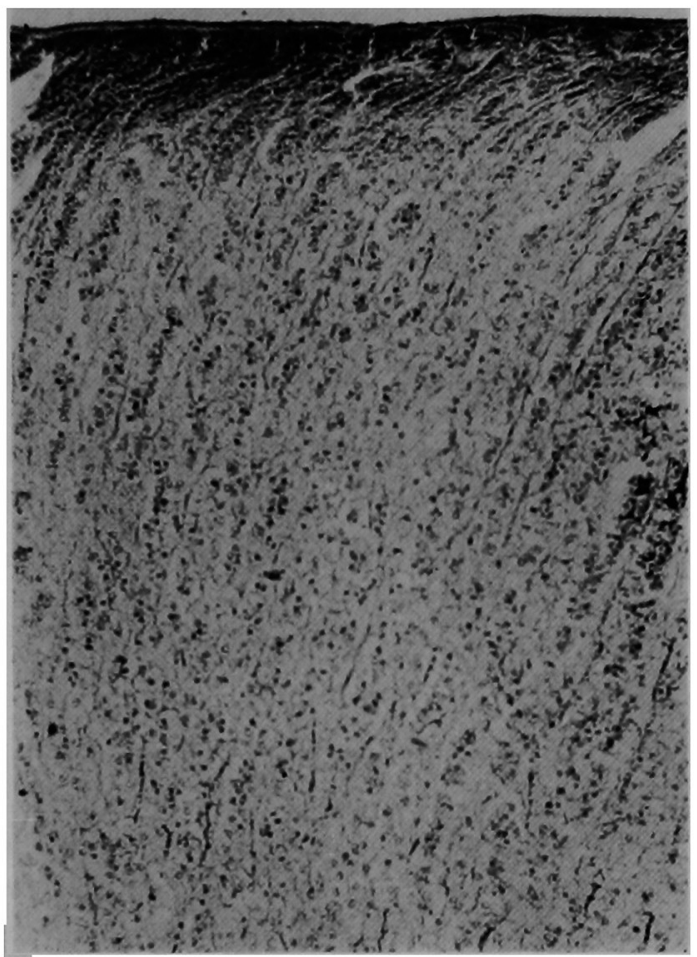

Fig. $1: 3 \boldsymbol{\beta}$-HSD in rat adrenal gland. Substrate-dehydroepiandrosterone.

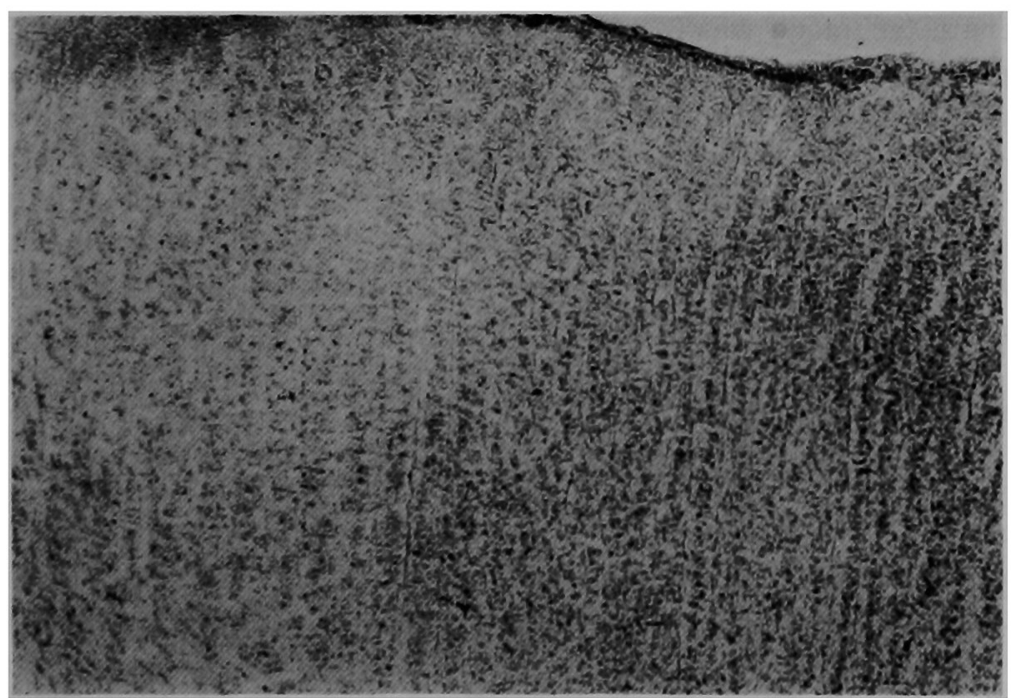

Fig. $2: 3 \beta$-HSD in rat adrenal gland. Substrate-epiandrosterone,

In fresh-frozen sections, TPN and DPN were effective as co-factor in the same degree.

Other factors concerning this staining such as phenazine methosulfate, $\mathrm{KCN}$ 
and nicotinamide were also studied. Phenazine methosulfate and nicotinamide were known to increase the production of formazan, while $\mathrm{KCN}$ gave no marked changes of the formazan formation.

\title{
Biochemical Problems of the Enzyme Fixation for the Histochemical Demonstration of Phosphorylase.
}

\author{
Moriichi Uchida, Yukiharu Shiraishi and Tadao TAKeUchI. \\ Department of Pathology, Kumamoto University School of Medicine, Kumamoto.
}

An effort to determine the relationship of the soluble (so-called "lyo-") and tissue-bound ("desmo") components of phosphorylase was made in this paper, because of labile demonstration of this enzyme in the tissue sections without fixation after cryostat cut. The activity with AMP was quantitatively measured by the Sutherland's modified method. The heart muscle of dogs was used in this experiment.

The total phosphorylase activity in the fresh wet tissue by use of the homogenate technique was 37.1 units $(37.1 \mathrm{mg} \mathrm{P} / \mathrm{g})$. The activity in the lyofraction formed 69 per cent (25.7 units) of the total, while the desmoenzyme activity showed only 26 per cent ( 9.6 units). On the other hand, when the cryostat cut sections were completely dried at room temperature for four hours

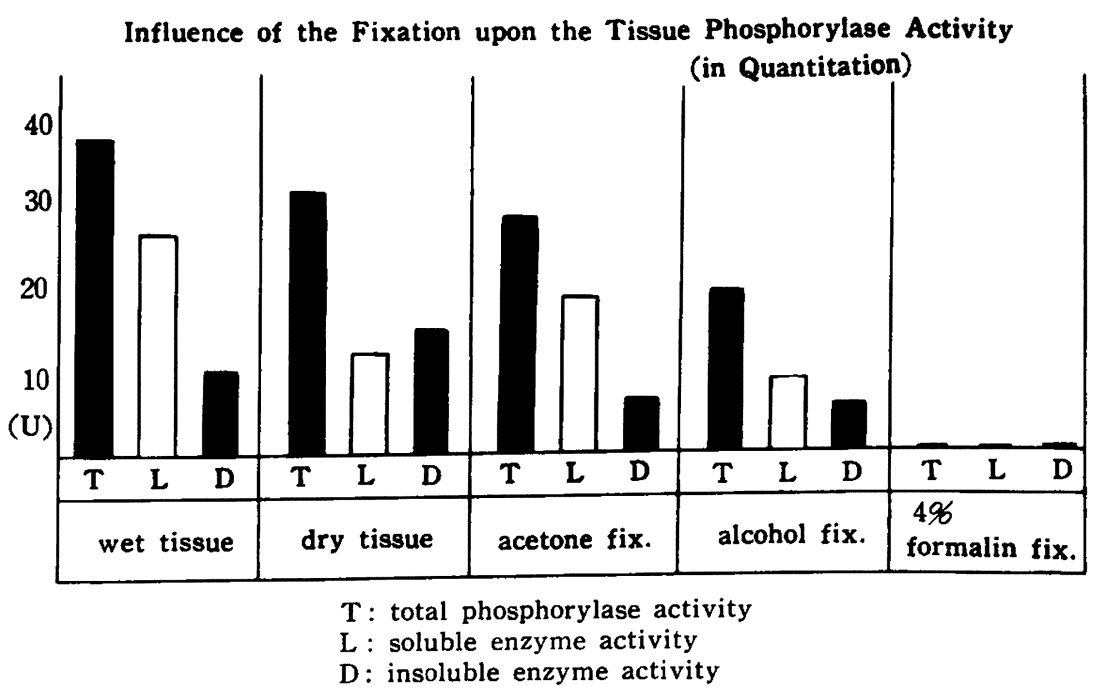

